\title{
SURFACE-MOUNTED PERMANENT MAGNET BRUSHLESS MOTOR SENSORLESS CONTROL BASED ON EMF EXTRACTION AND EXTENDED KALMAN ESTIMATOR
}

\author{
O. M. Arafa ${ }^{(1)}$, O. A. Mahgoub ${ }^{(2)}$, M. N. Eskander ${ }^{(3)}$ \\ ${ }^{1,3 j}$ Electronics Research Institute, El-Tahrir street, Dokki, Cairo, Egypt \\ ${ }^{2)}$ Department of Electrical Power \& Machines, Cairo University Giza, Egypt
}

\begin{abstract}
In this paper, a powerful method for sensorless control of permanent magnet brushless motors is explained. The method doesn't include any modification to the classical construction of such motors and can be easily integrated within the drive electronics. It is applicable to both motor types, i.e. those with sinusoidal or trapezoidal back EMF. The method is independent of excitation current profile. It is also virtually independent of system parameters. The back EMF waveform is extracted on-line using simple and integration-free algebraic expression through a novel measurement setup. It is then used within the corrective phase of an extended Kalman filter (EKF) to enable accurate estimation of the rotor position and speed. The method functionality is tested by simulation for sinusoidal back EMF motor with sinusoidal and quasi-square excitation as representative cases. Simulation results show a good performance at very low speeds.
\end{abstract}

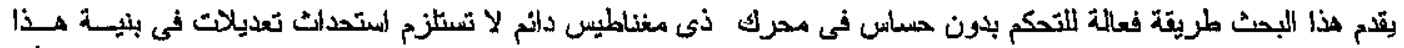

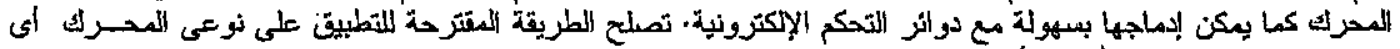

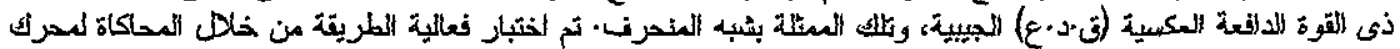

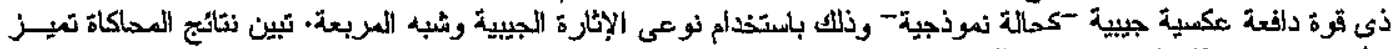

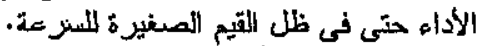

Keywords: Sensorless control, Extended Kalman filter, Pormanent magnet synchronous motor, Brushless DC motor.

\section{INTRODUCTION}

Permanent magnet brushless motor (PMBM) is widely used as a basic element in high performance servo drive systems due to its high efficiency and torque density. Recently, a lot of research has been focused on the sensorless control of PMB motors, which normally operate using position and speed sensors. Two different types of surface-mounted (SM) PMBM are available, the sinusoidal back EMF type and the trapezoidal back EMF type. The first type is frequently referred to as permanent magnet synchronous motor (PMSM) and the second type is frequently referred to as brushless $\mathrm{DC}$ motor (BDCM). Sensorless control is an attractive solution to mechanical sensor problems, which include extra cost, wiring complexity, reduced reliability and demand for additional mounting space. The authors of this study believe that space limitations [1], incompatibility with some harsh working environment (e.g. inside refrigerant compressors) [2] and weight reduction considerations (e.g. aviation industry) represent the most apparent motives behind sensorless control research efforts. This is particularly true if we consider one of the potential fields of application of such motors, i.e. computer disk drives, where miniaturization and reliability come as top design priorities [3].

Sensorless control schemes for surface-mounted (SM) PMBM are mostly depending on direct or indirect back EMF detection since the other schemes employing inductance changes with rotor position are applicable only to interior magnet (IM) PMBM [3].

Previous works developed sensorless control schemes for BDCM based on direct EMF detection including [3-6] where EMF zero crossings provide position and speed information six times the number of pole-pairs every rotor cycle. The major drawback of such technique is the availability of speed information once every one sixth of the electrical cycle. This further degrades the drive's dynamic performance, which is readily suffering from large torque dips during commutation intervals. The same concept equally applies to the alternative method proposed in [1], which extracts the rotor position information from the conduction state of the inverter's free wheeling diodes. 
Trying to provide continuous position and speed information, Hamid A. Toliyat, et al suggested a novel scheme for indirect back EMF detection [7], [8]. The scheme is well functioning even at very low rotational speeds. However, it involves some modification to the classical structure of such motors regarding the coils' arrangement and the number of leads to be brought out of the motor. This is generally inconvenient for automatic winders; also phasesplitting requires a non-standard production of PMSM. The scheme is therefore restricted to customdesigned PMSM and can't be extended to the case of BDCM as well.

Serving the same target, a comparative study [10] have proven that substantial improvement in BDCM performance can be realized by adopting an extended Kalman filter (EKF) algorithm utilizing the open phase voltage measurement in its correction mechanism [9]. According to the proposed scheme, continuous position and speed estimation is possible. Unfortunately the implementation is dedicated to BDCM only and can't be extended to PMSM.

Silverio Bolognani, et al [11] introduced a highperformance sensorless PMSM drive based on EKF covering most crucial aspects like the impact of motor parameter changes on performance, and the randomness of sense of rotation upon startup etc. The proposed remedies are excellent and well applicable in our current case; therefore we will not repeat them. The EKF resides on a motor model that includes both electrical and mechanical system dynamics in its state equation and on two current measurements in its output equation. The inclusion of electrical system dynamics requires short filter cycle comparable to the electrical time constant, hence fast digital signal processing is a must for successful implementation. Also the four by four matrices manipulation due to the selected model further increases the hardware speed requirement. After all, the scheme is applicable only to PMSM.

Parallel to the benefits gained from inductance dependence on rotor position in sensorless control of IM PMBM, It is quite normal to make benefits from its independence of rotor position in sensorless control of SM PMBM. In this study we try to make benefits from the special construction of PMBM as it is, thus avoiding any structural modifications. A unified and general approach for position and speed estimation capable of handling both PMSM and BDCM is introduced. The proposed technique is also independent of the excitation current profile since PMSM can be excited using sinusoidal or rectangular current waveforms. A new formulation of EKF is proposed which deals only with mechanical system dynamics. The measurement model of the EKF is based on a newly proposed method for indirect back EMF detection.

\section{SM PMBM MODEL}

The effective air gap of SM PMBM is virtually large and uniform due to the considerable magnet depth in the radial direction and due to the fact that magnet materials has permeability close to that of free air [12]. The large air gap helps maintain the magnetic circuit virtually free from saturation and keeping the winding's inductance nearly constant and independent of rotor position and excitation current.

On the other hand, PMBMs used in servo applications never operate without current control loop, this further support our assumption regarding the linearity of magnetic circuit since we can enforce control rules that keep current within magnetic circuit's linearity limits. A quite general voltage equation of both surface-mounted PMBM motor types can be written as:

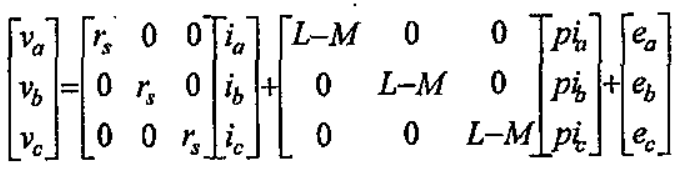

where:

$v_{a}, v_{b}, v_{c} \quad$ stator terminal voltage of phases $\mathrm{a}, \mathrm{b}$ and $\mathrm{c}$, respectively in Volt.

$i_{a}, i_{b}, i_{c} \quad$ :currents of phases $\mathrm{a}, \mathrm{b}$ and $\mathrm{c}$, respectively in Ampere.

$p \quad$ :differential operator.

$L \quad$ :self-inductance per phase in Henry.

$M \quad$ imutual inductance between two phases in Henry.

$e_{a} e_{b}, e_{c} \quad$ iback EMF of phases $\mathrm{a}, \mathrm{b}$ and $\mathrm{c}$, respectively in Volt.

$r_{s} \quad$ stator resistance per phase in $\mathrm{Ohm}$. $\cdots$

There are two complementary equations of the motor model. They are the mechanical system dynamics and the back EMF equation and we will explore them later to avoid repetition.

Equation (1), which is valid under the condition of isolated neutral point, shows that the three motor phases seem as if they were three independent coils of equivalent inductance (L-M) (though in fact they mutually interact through the mutual inductance term). The three windings are subject to the flux of a rotating magnet which induces a back EMF of value $e_{p h}$.

\section{BACK EMF DETECTION}

\section{A) THEORY}

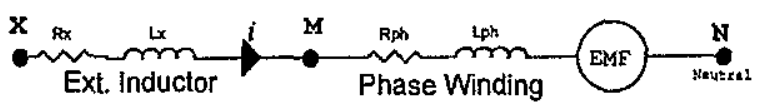

Fig. 1 External small inductor connected to each phase of PMBM 
In the circuit shown in Figure 1, let the circuit part from $M$ to $N$ represents one of these three independent coils, a small external coil is placed between points $X$ (inverter's feeding point) and $M$ (motor terminal). Writing the equations of voltage drop between points $M, N$ and $X, M$ respectively:

$$
\begin{aligned}
& V_{M N}=R_{p h} \cdot i+L_{p h} \cdot d i / d t+e \\
& V_{x M}=R_{x} \cdot i+L_{x} \cdot d i / d t
\end{aligned}
$$

\section{where:}

$V_{M N}$ : per phase voltage drop from $\mathbf{M}$ to $\mathbf{N}$ in Volt.

$V_{X M}$ : per phase voltage drop from $\mathbf{X}$ to $\mathbf{M}$ in Volt.

$e \quad$ : rotational back EMF generated due to permanent magnet excitation in Volt.

$L_{p h} \quad$ : equivalent inductance $(L-M)$ of the phase winding in Henry.

$R_{p h} \quad$ : resistance $\left(r_{s}\right)$ of the phase winding in Ohm. $L_{x} R_{x}$ : inductance in Henry and resistance in $\mathrm{Ohm}$ of the external inductor.

$i \quad$ : phase current in Amp.

Solving the two previous equations for e yields:

$$
e=-\frac{L_{p h}}{L_{x}} V_{x u}+\left(\frac{R_{x} L_{\rho h}}{L_{x}}-R_{p h}\right) i+V_{s N}
$$

The previous equation can be applied for each motor phase independently. Regarding current measurement needed for equation (4), current control loop normally needs measurement of two-phase current, hence naturally provides the current measurement required by the proposed method.

\section{B) CONDITIONS OF VALIDITY}

Equation (4) can be used on line to calculate the instantaneous value of the back EMF (with reasonable degree of accuracy) if the following presumptions could be satisfied:

- The motor magnetic circuit is operated in the linear region with no saturation and hence the inductance of the windings is fixed and not function of currents. Recall that SM PMBM easily satisfies this condition.

- Inductive voltage drops across windings' inductance are too large compared with the resistive voltage drops, this is also real when current shaping is done using a high frequency switching process. This condition is to ensure that the method is virtually insensitive to resistance variation due to thermal drift.

- Differential voltage measurement (and associated processing) at the selected points in Figure 1 is simultaneous and fast enough relative to the current control switching frequency. Using proper $\mathrm{A} / \mathrm{D}$ configuration with ample bandwidth can satisfy this requirement.

- The external inductors -which can be mounted on the inverter's PCB- are to be made identical to each other with low-loss core, with constant inductance, minimum resistance and should have zero-mutual inductance. These conditions can also be met by a careful design.

\section{SYSTEM STATE EQUATION}

\section{A) CONTINUOUS FORM}

EKF requires a dynamic system model (a state equation) [13]. The state equation for the proposed implementation will involve mechanical system dynamics with electromagnetic developed torque and load torque considered as deterministic system input. The state equation is written in continuous statespace form as follows:

$$
\left[\begin{array}{c}
\dot{\omega}_{r} \\
\dot{\theta}_{r}
\end{array}\right]=\left[\begin{array}{cc}
-\frac{B}{J} & 0 \\
1 & 0
\end{array}\right]\left[\begin{array}{c}
\omega_{r} \\
\theta_{r}
\end{array}\right]+\left[\begin{array}{c}
\frac{P}{2 J} \\
0
\end{array}\right]\left(T_{e}-T_{L}\right)
$$

where:

$$
\begin{array}{ll}
\omega_{r}, \theta_{r} & \begin{array}{l}
: \text { rotor's electrical speed in } \mathrm{rad} / \mathrm{s} \text { and the } \\
\text { rotor's electrical position in rad, } \\
\text { respectively. }
\end{array} \\
B \quad \begin{array}{l}
: \text { Damping friction coefficient (rotor and } \\
\text { load) in N.m.s/rad. }
\end{array} \\
\begin{array}{l}
\text { : Inertia of the rotating mass (rotor and } \\
\text { load) in N.m.s } \mathrm{s}^{2} / \mathrm{rad} .
\end{array} \\
T_{e}, T_{L} \quad \begin{array}{l}
: \text { Number of poles. } \\
\text { : electromagnetic developed torque and } \\
\text { load torque in N.m, respectively. }
\end{array}
\end{array}
$$

The electromagnetic torque $T_{e}$ is given by :

$T_{e}=\lambda_{m}\left(\frac{P}{2}\right)\left(\frac{3}{2} i_{a} \cos \theta_{r}+\frac{\sqrt{3}}{2}\left(i_{b}-i_{c}\right) \sin \theta_{r}\right)$

where $i_{a} i_{b}$ and $i_{c}$ are the three phase currents.

\section{B) DISCRETIZATION}

The continuous state space representation of the form $\dot{\mathbf{x}}=\mathbf{A x}+\mathbf{B u}$ can be described by the following difference equation [14]:

$$
\mathbf{x}((k+1) T)=A^{\prime}(T) x(k T)+B^{\prime}(T) u(k T)
$$

where $A^{\prime}(T)$ and $B^{\prime}(T)$ are dependent on sampling period $T$, once $T$ is fixed, the matrices $A^{\prime}(T)$ and $B^{\prime}(T)$ are constant matrices: 


$$
\begin{aligned}
& A^{\prime}(T)=e^{\mathbf{A} T} \\
& B^{\prime}(T)=\left(\int_{0}^{T} e^{\mathbf{A} T} d t\right) \mathbf{B} \\
& \text { where: } \\
& e^{\mathbf{A} T}=L^{-1}\left[(s \mathbf{I}-\mathbf{A})^{-1}\right]
\end{aligned}
$$

$\mathrm{L}^{-1}$ is the inverse Laplace transformation. Therefore, discretization of equation (5) yields:

$$
\begin{aligned}
& {\left[\begin{array}{l}
\omega_{r}(k+1) \\
\theta_{r}(k+1)
\end{array}\right]=\left[\begin{array}{cc}
e^{\frac{-T}{\tau}} & 0 \\
\tau\left(1-e^{\frac{-T}{\tau}}\right) & 1
\end{array}\right]\left[\begin{array}{l}
\omega_{r}(k) \\
\theta_{r}(k)
\end{array}\right]+} \\
& {\left[\begin{array}{c}
\frac{P \tau}{2 J}\left(e^{\frac{-T}{\tau}}-1\right) \\
\frac{P}{2 J}[\tau \\
\left.\tau+\tau^{2}\left(e^{\frac{-T}{\tau}}-1\right)\right]
\end{array}\right]\left(T_{0}-T_{L}\right)}
\end{aligned}
$$

\section{SYSTEM OUTPUT (MEASUREMENT) EQUATION}

EKF inspires its corrective action from a real measurement and an equation that relates this measurement to system state [13], we will consider the three-phase back EMF as system's output that we measure. This output $\mathrm{z}$ is in general given by a nonlinear equation of the form:

$$
\mathbf{z}_{k}=h\left(\mathbf{x}_{k}, \mathbf{v}_{k}\right)
$$

where :

$\mathbf{z}_{k}$ : back EMF of the windings $\mathrm{a}, \mathrm{b}$ and $\mathrm{c}$ detected at step $\mathrm{k}\left[\begin{array}{lll}e_{a k} & e_{b k} & e_{c k}\end{array}\right]^{T}$.

$\mathbf{x}_{k} \quad:$ State variables $\left[\omega_{r}(k) \quad \theta_{r}(k)\right]^{T}$.

$\mathbf{v}_{k}$ : Random variable representing the measurement noise. It is assumed to be of normal probability distribution with zero mean and with covariance $\mathbf{R}$ i.e. $p(\mathbf{v}) \sim N(0, \mathbf{R})$.

For PMSM type (regardless the type of excitation used i.e. sinusoidal or block-type), neglecting for a while the measurement noise term in equation (13), the measurement equation $h\left(\mathbf{x}_{k}, \mathbf{v}_{k}\right)$ is given by:

$$
\left[\begin{array}{l}
e_{a} \\
e_{b} \\
e_{c}
\end{array}\right]=\lambda_{m}^{\prime} \frac{d \theta_{r}}{d t}\left[\begin{array}{c}
\cos \theta_{r} \\
\cos \left(\theta_{r}-\frac{2}{3} \pi\right) \\
\cos \left(\theta_{r}+\frac{2}{3} \pi\right)
\end{array}\right]
$$

where:

$i_{m} \quad$ : the permanent magnet flux in $\mathrm{Wb}$ or the back EMF constant in volt.s/rad. Application of EKF requires linearizing equation (13).
According to the known linearization procedure, the Jacobean matrix $\mathrm{H}$ is given as follows:

$$
\mathbf{H}=\lambda_{m}^{\prime}\left[\begin{array}{cc}
\cos \theta_{r} & -\omega_{r} \sin \theta_{r} \\
\cos \left(\theta_{r}-\frac{2 \pi}{3}\right) & -\omega_{r} \sin \left(\theta_{r}-\frac{2 \pi}{3}\right) \\
\cos \left(\theta_{r}+\frac{2 \pi}{3}\right) & -\omega_{r} \sin \left(\theta_{r}+\frac{2 \pi}{3}\right)
\end{array}\right]
$$

At each time step, the elements of $\boldsymbol{H}$ should be updated with new values of $\theta_{r}$ and $\omega_{r}$.

\section{EKF ESTIMATION CYCLE}

EKF has an estimation cycle composed of two main phases, namely prediction phase and correction phase. The filter starts normally the prediction phase with an initial guess of state, then by its recursive structure, new estimates coming out of the correction phase are used to feed the prediction phase in the succeeding cycle.

\section{A) TIME UPDATE ("PREDICT")}

Project the state ahead in time

$\widetilde{\mathbf{x}}_{k}=\mathbf{A} \hat{\mathbf{x}}_{k-1}+\mathbf{B} \mathbf{u}_{k-1}$

where:

$\widetilde{\mathbf{x}}_{k} \quad:$ Prediction of the current state.

$\hat{\mathbf{x}}_{k-1}$. : Previous estimate of state. (On the first filter's cycle, this is the initial state guess).

$\mathbf{u}_{\mathrm{k}-1} \quad$ : system input given by equation (12).

Matrices $\mathbf{A}$ and $\mathbf{B}$ of equation (15) are given in equations (8) and (9) by A'and B' respectively, the components of the vector $\mathrm{x}$ are the rotor speed and position, respectively as shown in equation (12).

Project the state-prediction error covariance ahead in time

$\widetilde{\mathbf{P}}_{k}=\mathbf{A} \hat{\mathbf{P}}_{k-1} \mathbf{A}^{T}+\mathbf{Q}_{k-1}$

$\widetilde{\mathbf{P}}_{k} \quad$ : prediction of the covariance of state prediction error.

$\hat{\mathbf{P}}_{k-1} \quad$ : previous estimate of the covariance of state prediction error (to be guessed in the first filter's cycle).

$\hat{\mathbf{Q}}_{k-1} \quad$ : process noise covariance.

Process noise is modeled as a random number $\mathbf{w}_{k}$ with normal probability distribution of zero mean and of covariance $\mathbf{Q}$ where $p(\mathbf{w}) \sim N(0, \mathbf{Q})$

\section{B) MEASUREMENT UPDATE ("CORRECT")}

\section{Compute Kalman Gain}




$$
\mathbf{K}_{k}=\frac{\widetilde{\mathbf{P}}_{k} \mathbf{H}_{k}^{T}}{\mathbf{R}_{k}+\mathbf{H}_{k} \widetilde{\mathbf{P}}_{k} \mathbf{H}_{k}^{T}}
$$

where $\mathbf{H}_{k}$ is given by equation (14), $\mathbf{R}_{k}$ is the measurement noise covariance. The denominator of the right hand side of the previous equation is referred to as covariance of innovation. Although $Q$ and $\mathrm{R}$ in the above equations have the subscripts $\mathrm{k}, \mathrm{k}-$ 1 which means they can have different values over time, in this application they are assumed to be time irrelevant

Update estimate with measurement $\mathbf{Z}_{k}$

$$
\hat{\mathbf{x}}_{k}=\widetilde{\mathbf{x}}_{k}+\mathbf{K}_{k}\left(z_{k}-h\left(\widetilde{\mathbf{x}}_{k}, 0\right)\right)
$$

where:

$\hat{\mathbf{x}}_{k} \quad$ : current estimate of state.

$z_{k} \quad:$ the extracted value of the back EMF as given by equation (4) using the appropriate voltage and current measurements .

Update the state-prediction error covariance

$$
\hat{\mathbf{P}}_{k}=\left(\mathbf{I}-\mathbf{K}_{k} \mathbf{H}_{k}\right) \widetilde{\mathbf{P}}_{k}
$$

where:

$$
\begin{aligned}
& \hat{\mathbf{P}}_{k} \text { : current estimate of the covariance of state } \\
& \text { I } \text { prediction error. } \\
& \text { : unity matrix. }
\end{aligned}
$$

The values of the vector $\hat{\mathbf{x}}_{k}$ represent the best estimate of rotor speed and position that are used to feed the speed and current control loops.

\section{SIMULATION}

Figure 2 illustrates the flowchart of the PMBM simulation program. The program is running in MATLAB/SIMULINK environment. The particular type of the motor and of the applied excitation is fed to the program through an identification parameter to distinguish between two different excitation modes of the program:

1. sinusoidal excitation mode.

2. quasi-square excitation mode.

In block 3, the speed error fed to that block from the comparison of speed command and the EKF estimated speed is being processed by a PI controller whose parameters are fed to the program in block 1.
This process results in torque command, which is then used to calculate the peak amplitude of current command.

A formatting envelope inheriting the waveform of the excitation current (sinusoidal or rectangular according to running mode) with unity amplitude is generated in block 9 based on EKF rotor position estimation. This envelope is used to issue the current command by direct amplitude modulation that takes place in the multiplication block 4.

PI current control then issues the voltage command to the inverter. Inverter control defines the status of the inverter devices and hence the inverter/motor circuit topology.

The proper set of differential equation is then picked up as in block 7 to calculate the current derivatives. Numerical integration with reasonable fixed step size is then applied to the state derivatives to obtain current values (rotor speed and position are also obtained at this stage for the sake of comparison with EKF estimation results). Now motor currents are fed into block 8 where developed torque can be calculated and EKF is applied (Load torque is considered as disturbance). Speed and position estimation results from EKF are then used for closing the speed feedback loop and for creating excitation current template as per block 9 .

In the following figures the results obtained from simulation runs intended for testing the dynamic (transient) and steady state performance of the newly proposed method using this program, and applied on a PMSM motor, are introduced (Motor's parameters are listed in the appendix. section).

In these simulation runs, a no-load startup is initially allowed with a speed command of half the rated speed, and with zero initial rotor position. Then at $t=0.1333 \mathrm{sec}$ the full load torque is applied, and speed command is suddenly raised to full rated speed. Motor successfully accelerates to the full rated speed in a forward direction as commanded. At $\mathrm{t}=0.2666$, the speed is commanded to reverse and load torque is suddenly applied in the reverse direction. The motor decelerates to zero then accelerates in the reverse direction until rated speed is reached.

The developed torque diagrams show the acceleration periods where the torque value is within the limits imposed by the torque and current controllers until reaching the desired speed then drops off to the level required for load driving. Figure 3 (a) through Figure 3 (e) illustrates PMSM response with sinusoidal excitation. The case of PMSM with quasi-square type excitations is shown in Figure 4 (a) through Figure 4 (e). 
O. Arafa, O. Mahgoub, M. Eskander "Surface-Mounted Permanent magnet brushless ..."

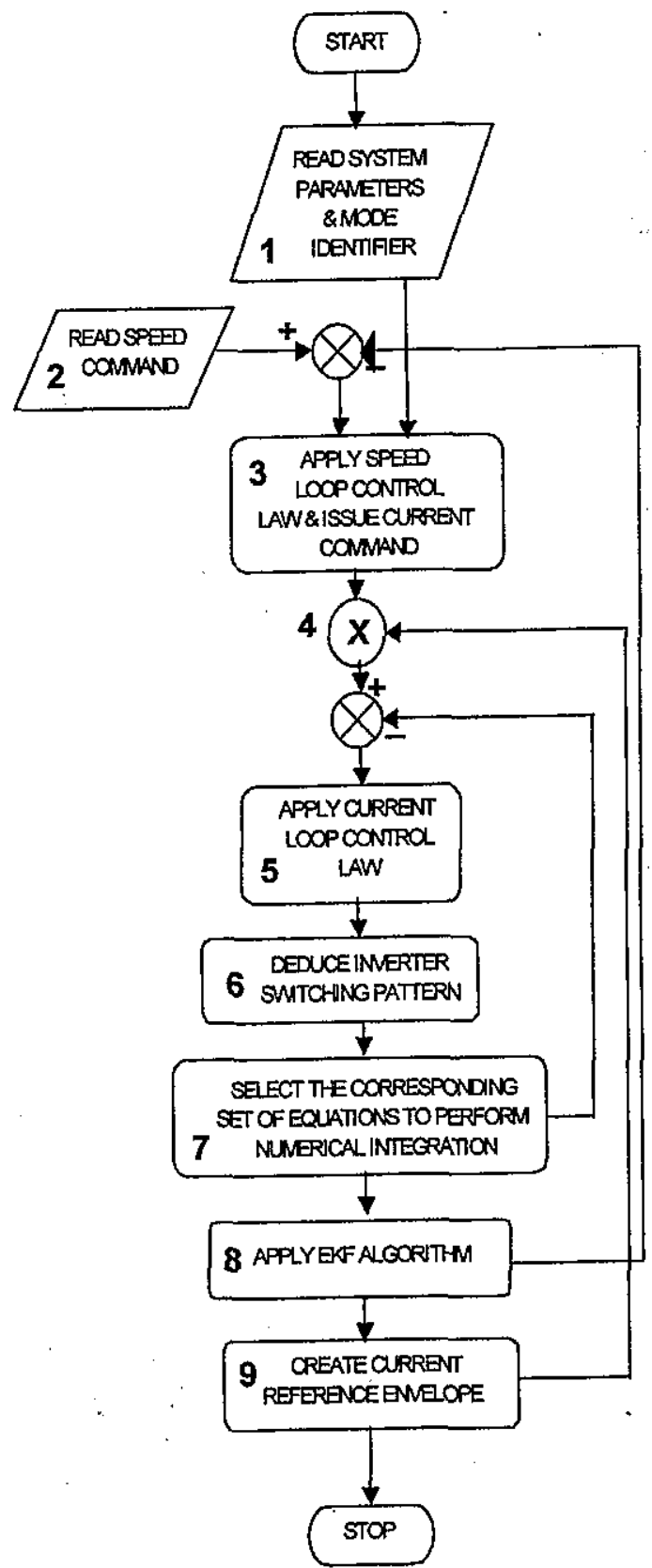

Fig. 2 Simulation program flowchart

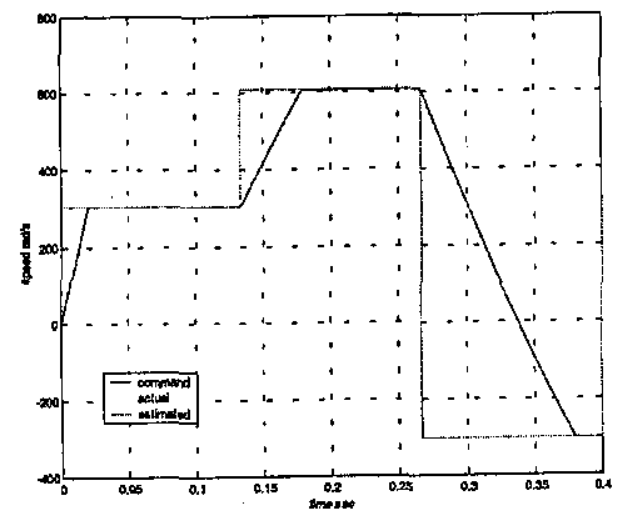

a) Motor speed profile
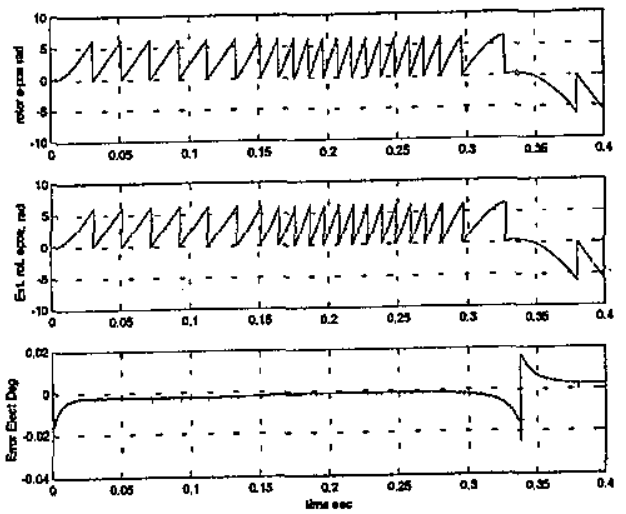

b) Electrical rotor position (Actual/estimated/error)

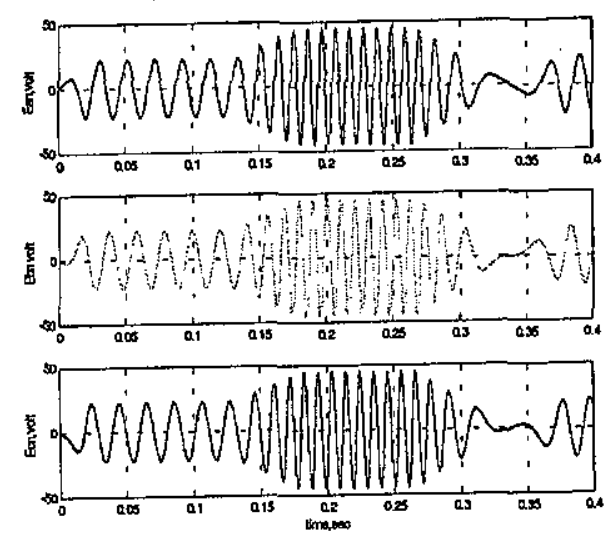

c) EMF detected by the proposed method (phase A,B,C)
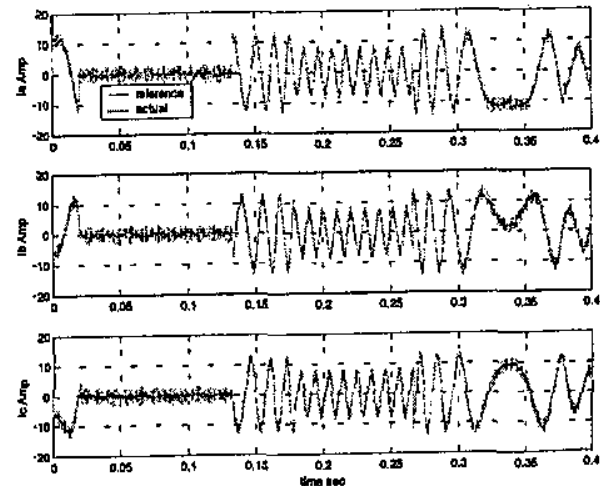

d) Rotor current (phases $\mathrm{A}, \mathrm{B}, \mathrm{C}$ )

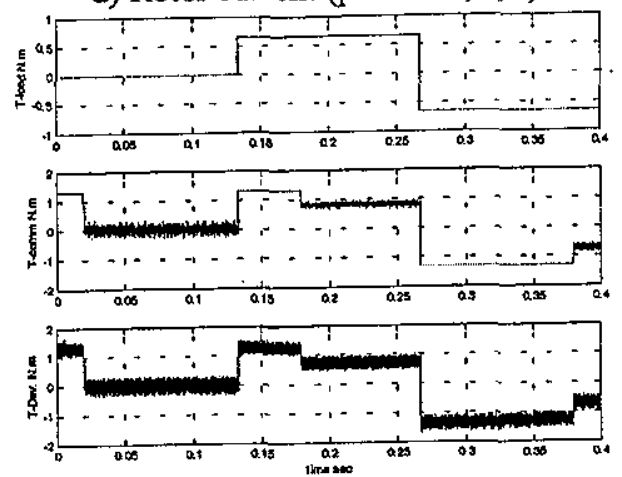

e) Motor torque profile (Load/Command/Developed) Fig. 3 PMSM performance curves with sinusoidaltype excitation 


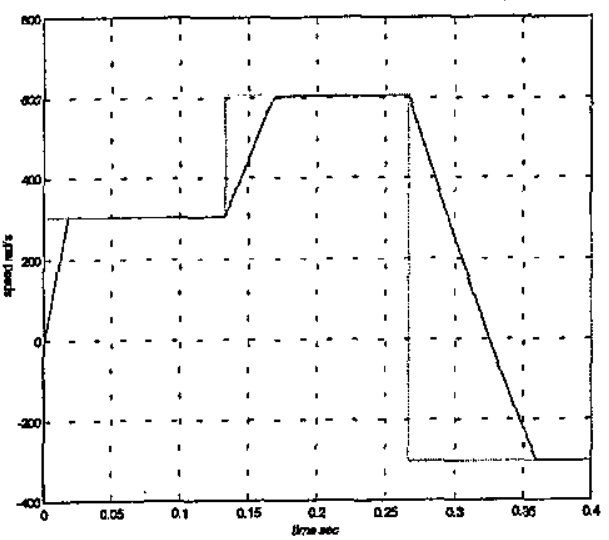

a) Motor speed profile
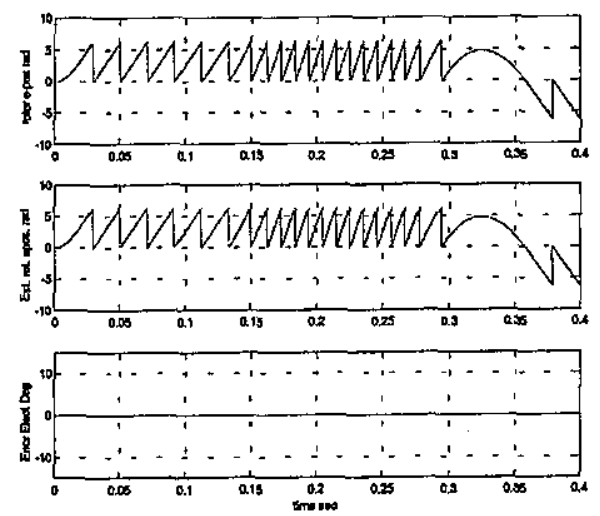

b) Electrical Rotor Position (Actual/Estimated/Error)
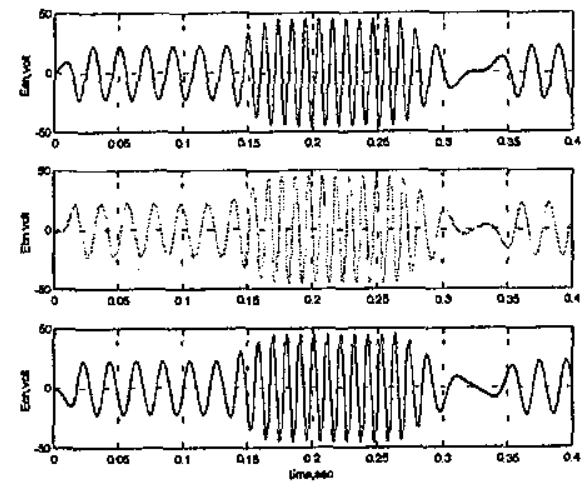

c) EMF detected by the proposed method (phase $\mathrm{A}, \mathrm{B}, \mathrm{C})$

$\mathrm{v}$

d) Rotor Current Waveforms (phase $\mathrm{A} / \mathrm{B} / \mathrm{C}$ )
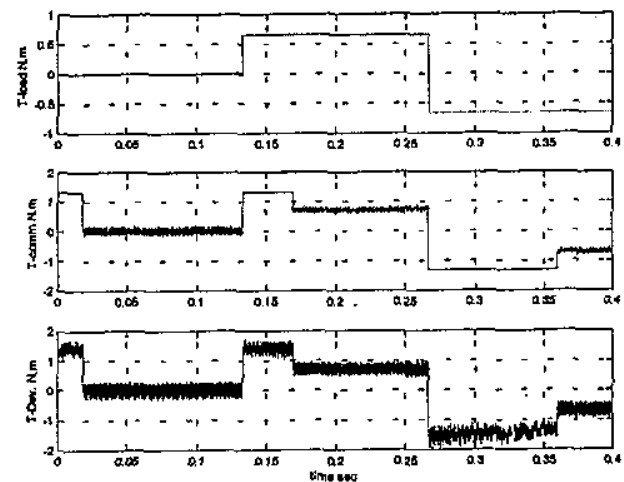

e) Motor torque profịle (Load/Command/Developed)

Fig.4 PMSM performance curves with quasi-square type excitation

\section{CONCLUSION}

The advantages obtained by using powerful stochastic filtering along with a unified approach for PMBM sensorless control is discussed in this paper. Based on the concept presented here, a single controller design can be realized for commercial use which is capable of providing superior control for both PMSM and BDCM. Parameters of motor and external coil are all what we need to apply the solution to a particular case. Power cable only is needed to connect the motor to the drive electronics, neither extra taps nor winding modifications are needed.

Using a state model based on mechanical system dynamics with its virtually large time constant, which allows for longer filter cycle, effectively reduces the intensive on-line arithmetic characterizing EKF. It also allows for reduced-size matrix manipulation (mostly $2 \times 2$ matrices) and thus further reduces the hardware speed requirement. This finally reduces the cost while still offering the performance quality provided by larger complex models.

The external inductor value should be minimized to reduce the voltage drop across it. The resolution of the A/D conversion is a critical factor in deciding the minimum acceptable value of the inductor.

\section{REFERENCES}

[1] Ogasawara S., Akagi H., "An Approach to Position Sensorless Drive for Brushless DC Motor", IEEE Transactions on Industry Applications, September/October, 1991, PP 928-933.

[2] Kinichi IIzuka, Hideo Uzuhashi, Minoru Kano, Tsunehiro Endo, 'Microcomputer Control for Sensorless Brushless Motor", IEEE 
Transactions on Industry Applications, Vol. IA21, No. 3, May/June 1985, PP 595-601.

[3] L. Cardoletti, A. Cassat, M. Jufer, "Sensorless Position and Speed Control of a Brushless DC Motor from Start-up to Nominal Speed" ,EPE Journal, Vol.2, No.l (1990), PP 25-34.

[4] D. M. Erdman, H. B. Harms, J. L. Oldenkamp, "Electronically Commutated DC motors for the Appliance Industry", IEEE-IAS Conf. Rec., Oct. 1984, PP 1339-1345.

[5] N. A. Demerdash, T. W. Nehl, E. Maslowski, "Dynamic modeling of brushless dc motors in electric propulsion and electromechanical actuation by digital techniques", IEEE IAC Conference Rec., 1980, PP 570-579.

[6] P. Zimmermann, "Electronically commutated D.C. drives for machine tools", Motorcon Proceedings, 1982, PP 69-83.

[7] Deepak S. Shet, Hamid A. Toliyat, Thomas A. Nondahl, "Position Sensorless Control of Surface Mount Permanent Magnet AC (PMAC) Motors at Low Speeds", Proceeding of the American Control Conf. San Diego, California, June 1999 PP 2141-2142.

[8] Khawaja M. Rahman, Hamid A. Toliyat, "Sensorless Operation of Permanent Magnet AC (PMAC) motors with Modified Stator Windings", Industry Application. Conf., 31st Annual IAS Meeting, 1996, vol.1 PP 326-333.

[9] Paul Kettle; Aengus Murray, Finbarr Moynihan, "Sensorless Control of a Brushless DC Motor using an Extended Kalman Estimator", PCIM'98 Intelligent Motion. Nurenberg, May 1998, Proceedings, PP 385-392 .

[10] Petre Minciunescu, Tom Flint, Finbart Moynihan, Paul Kettle, "Sensorless Control of Brushless DC Motors Using Extended Kalman
Filter Estimator and Back EMF Integration Algorithm: A Comparison", Embedded Control Systems, Analog Devices Inc.

[11] Silverio Blognanai, Mauro Zigliotto, Marco Zordan, "Extended-Range PMSM Sensorless Speed Drive Based on Stochastic Filtering", IEEE Transactions on Power Electronics, Vol.16, No.1, Jan 2001, PP 110-116.

[12] T.W. Nehl, N.A. Demerdash, F.A. Fouad, "Impact of Winding Inductance and Other Parameters on the Design and Performance of Brushless DC motors", IEEE Transactions on Power Apparatus and Systems, Vol. PAS-104, No. 8, August 1985, PP 2206-2211.

[13] Peter S. Maybeck, "Stochastic Models, estimation, and Control" Volume 1, Academic Press Inc (AP) New York, 1979.

[14] Katsuhiko Ogata, "Modern Control Engineering", Prentice-Hall Engineering-4th Edition, 2001.

\section{APPENDIX}

The parameters of a 400-watt SM PMSM motor used in simulation are listed below:

$\mathrm{R}$ (Resistance per phase) $=0.560 \quad \Omega$

$\mathrm{L}^{\prime}=\mathrm{L}_{\mathrm{s}}-\mathrm{L}_{\mathrm{M}} \quad=5.945 \mathrm{e}-4 \quad \mathrm{H}$

$\lambda_{m}^{\prime}$ (Back EMF const) $=0.073 \mathrm{~V} / \mathrm{rad} / \mathrm{s}$

$\mathrm{J}$ (Inertia) $\quad=831 \mathrm{e}-7 \mathrm{Kgm}^{2}$

$\mathrm{B}$ (friction coeff.) $\quad=1.139 \mathrm{e}-4 \mathrm{~N} \cdot \mathrm{m} . \mathrm{s} / \mathrm{rad}$

$\mathrm{P}$ (Number of poles) $=2$ pole

Rated continuous torque $=660 \mathrm{e}-3$ N.m.

Rated Speed $\quad=5800$ R.P.M.

Rated power $\quad=400 \mathrm{~W}$

Rated Current $\quad=4.51 \mathrm{~A}$ 